\title{
Surveying Industrial Roles in Open Source Software Development
}

\author{
Øyvind Hauge, Carl-Fredrik Sørensen, Andreas Røsdal \\ Norwegian University of Science and Technology (NTNU), 7491 \\ Trondheim, Norway
}

\begin{abstract}
Industry uses Open Source Software (OSS) to a greater and greater extent. We have defined four industrial OSS roles; OSS provider, OSS integrator, OSS participant and Inner Source Software (ISS) participant. Based on these four roles we have performed a survey in the ITEA COSI project. We provide initial answers to what motivates companies to undertake these roles, what are the advantages and challenges of undertaking them, and which development practices they use while undertaking these roles.
\end{abstract}

Key words: Open Source, Industry, Roles, Survey, Motivations, Development Practices

\section{Introduction}

The cost of producing software from scratch goes hand in hand with the steadily increasing size and complexity of the software. Reuse of standard components has been seen as one solution to keep costs down. Reusable components have been developed in-house or acquired from other vendors.

OSS provides quality software, enables new ways of developing software, and makes new business strategies possible. OSS can be important in the battle against constantly larger and more complex software. Several major industrial actors like Sun Microsystems, Oracle, IBM, and Novell, have already started to benefit from OSS.

The entry of industry into the OSS field opens up a new research arena. The ITEA COSI project wants to increase the understanding of how industry can benefit from OSS. As part of the ongoing work in the ITEA COSI project we have performed a survey of current OSS development practices in parts of the European software industry. The survey gave several interesting indications. The availability of OSS is perhaps the most important reason behind use of OSS. The main advantages for a company having an OSS product come from, value added by supplementary products and community innovation. Attracting and supporting an OSS community requires hard work and there are challenges related to community contributions.

We start by presenting the four industrial OSS roles and the applied research method before we present our results and sum up with a discussion and conclusions.

Please wise the following format when citing this chapter:

Hauge, Ø., Sørensen, C.-F., Røsdal, A., 2007, in IFIP International Federation for Information Processing, Volune 234, Open Source Development, Adoption and Innovation, eds. J. Feller, Fitzgerald, B., Scacchi, W., Sillitti, A., (Boston: Springer), pp. 259-264. 


\section{Related work and Industrial Roles}

Our literature survey did not discover many empirical studies of industrial OSS involvement. However, examples can be found e.g. [1-5].

We want to highlight the need for more varied and reproducible empirical research. The majority of the publications we found were case studies or experience reports which are hard to reproduce. The work is in many cases performed in only one setting, most often in a non-industrial setting.

Based on literature and conversations with the industrial partners of the ITEA COSI project we defined four industrial roles: OSS Provider, OSS Integrator, OSS Participant, and Inner Source Software (ISS) Participant.

An OSS provider is a company which controls the code base of an OSS product. MySQL, Trolltech, and Sun Microsystems are some examples. The OSS integrator is a company which, uses OSS components in their products or build their products on top of OSS infrastructure. The OSS participant is a company actively interacting with one or more OSS projects. IBM and SUN are for instance participating in the development of the Apache DB. The ISS participant is a company participating in an inter department or inter company collaborative development using OSS development practices.

\section{Research Method}

In the first phase of the ITEA COSI project, we wish to create a baseline description of the industrial OSS related development. The following questions were based on a literature review and in conversations with project partners: Why do industrial actors undertake the four OSS roles? What are the advantages and challenges related to undertaking them? Which development practices are used in these roles?

Based on these questions, we created an interview guide which was used in semistructured interviews with Norwegian COSI partners. The interviews were performed at the offices of the industrial partners and all of them were recorded and later transcribed.

We interviewed two developers in company A, one developer in company B, and one developer and one CEO in company C. Company A is a small company which uses OSS in their development. Company B is a medium sized consulting company delivering services and products based on OSS. Company $\mathrm{C}$ is a medium sized company which provides an OSS product.

The interview guide and the results from the interviews were used as a basis for a web-survey. The survey had one part for each OSS role.

The ITEA COSI project consists of big companies from telecom and embedded software, but also smaller and more traditional software companies. Selection of the respondents was because of the composition of the project, unfortunately out of our hands. We distributed the survey to the all of the project partners and encouraged 
them to respond at least once. The companies selected their respondent(s) themselves and we received the following number of responses; OSS provider: 3, ISS participant: 6, OSS participant: 6 and OSS integrator: 9, in total 24 responses.

\section{Results}

OSS providers are motivated to release their products as OSS of several factors. The community can perform testing and provide new functionality, bug-fixes, bugreports, and translations. This may enhance the functionality and increase the quality of the product. The community members may contribute to the innovation of the product in form of new ideas and new requirements. They can also provide supplementary products and services.

Releasing a product as OSS is a way to make it available to a large user group. If the community is satisfied with the product, it will most likely share its experiences with others and thereby give the OSS provider free marketing and increased publicity.

Increased value, availability and publicity, boost the possibility of attracting new users. This is important because many industrial OSS providers sell services related to their OSS products. The more users, the more potentially paying customers and the more likely it is that someone will contribute to the development of the product.

We believe that the innovation and the supplementary products and services which increase the value of the product are more important than code contributions. This is because the Oss provider has to review contributions in form of code, requests, and opinions.

Maximizing community contributions and reducing the work related to these contributions is one of the challenges an OSS provider faces. Attracting a community is another major challenge for an OSS provider and according to our respondents, hard work.

It is important to offer the community a piece of quality software they need, infrastructure to support the community, enough documentation and information to get the community members going and to make them feel involved. However, it is important not to involve the community too much because involvement will create overhead and delays.

The OSS integrator is motivated by the low purchase price of the OSS products. Perhaps even more important is the high availability of OSS. Standard compliance was also mentioned as a reason why people use OSS.

Many OSS products are available through project web sites containing documentation, forums and mailing lists, bug and feature trackers, road maps, developer info and so on. The honesty about the true status of the OSS product and the availability of information make it easier for the OSS integrator to understand and evaluate it.

OSS components are primarily selected through informal processes. The OSS integrator discovers a need for a component. He forms an initial idea of what the 
software should do. Based on these initial requirements he performs an informal search to create a long-list. This long-list is later reduced to a short-list. The components on the short-list are tested or evaluated closer before one product is selected.

The candidate components may be found through many sources; prior experience, friends or co-workers, request for help on forum or mailing-list, searches in OSS portals or search engines. Search engines are used to find both single components and comparisons of several components.

Missing functionality, incompatible licenses, unfamiliar programming languages, lack of stable releases, no activity in community, bad or no reputation, and absence of documentation, are easy-to-check evaluation criteria. To evaluate the components further the developer may subscribe to mailing lists, study documentation, perform code reviews, and test the software in a small prototype. Plans and roadmaps, compatibility to other software, standard compliance, reputation of the product and the provider, the development process used in the community, and support from community or a commercial provider, were all mentioned as evaluation criteria in this process. This evaluation was mostly informal but some respondents reported that they used checklists.

The OSS integrator is faced with some challenges. There are vast numbers of OSS available out there and finding quality products can be hard.

By changing the source code of the OSS products he uses, the OSS integrator is left with two choices: He can keep the changes to himself or feed the changes back into the product. Convincing the OSS project to include these changes can be hard. If he is unable to make the OSS project include his changes he has to maintain this code himself. This could be time-consuming and it may lead to problems with new releases of the OSS.

Most of the OSS participants could not surprisingly be classified as active or passive users. They provide occasional bug fixes and requirements, subscribe to mailing-lists, read news, and primarily use the software.

The respondents were overall satisfied with the OSS products, their communities, information from the community, and their relationship with the community. However, they acknowledged that they would have been able to influence the community more through increased participation.

Participation as a company was not surprisingly rooted in the need for the product. Learning was also mentioned as one important motivation for some companies. On the individual level learning, idealism, and personal interest in the product were mentioned as the most important factors.

The participants in ISS development use some development practices often used in OSS development. The use of e-mail and mailing list was due to the distributed development quite extensive.

To provide the participating developers a shared view of the code, code repositories were used. These repositories were controlled by gatekeepers or module owners. Based on the code base, several pre-releases of the software were made 
available to give the users an early impression of the product and to allow the users to provide feedback to the developers.

Some of the respondents reported saved development effort and maintenance effort due to ISS cooperation.

\section{Discussion and conclusions}

In the section about related work we requested more and more varied empirical research related to industrial OSS involvement. We are aware of some of the limitations of our own work and we will discuss some of these here.

The survey was intended to be a baseline for the companies in the ITEA COSI project. The selection of respondents was done from this population and we cannot claim that our results are valid for other populations.

The number of respondents was unfortunately quite low. The selection of respondents was done by convenience sampling. We were, due to the sampling method, unable to control mortality rates and drop out rates for the questionnaire. These factors reduce the internal validity and the statistical validity of the survey.

We have however increased the validity through interviews with some of the respondents and through expert review. We have presented the results to the ITEA COSI project and to several of the respondents. None of them gave us any indications that the results were flawed.

We believe that our work is a step on the way to understand how industry can benefit from OSS products and development methodologies. The survey has given us initial ideas of what motivates companies to undertake the four roles OSS provider, OSS integrator, OSS participant, and ISS participant. Furthermore, we have described some of the advantages and challenges related to undertaking these roles. At last we have started to describe some of the processes and practices used by these roles.

The work of answering the initial questions about motivations, processes, advantages and challenges are by far not completed. We will continue this work and a second version of the survey is under development. This survey will be distributed to a larger European population through ITEA.

\section{Acknowledgement}

The Norwegian COSI is sponsored by the Norwegian Research Council's IKT2010 program. The COSI project is part of the ITEA 2 program. 


\section{References}

1. W-G. Bleek, M. Finck, and B Pape, Towards an Open Source Development Process? Evaluating the Migration to an Open Source Project by Means of the Capability Maturity Model, Proceedings of the First International Conference on Open Source Systems, Genova, Italy, 37-43 (2005)

2. C. Jensen and W. Scacchi, Collaboration, Leadership, Control, and Conflict Negotiation and the Netbeans.org Open Source Software Development Community, Proceedings of the 38th Annual Hawaii International Conference on System Sciences, 196b-196b, (2005).

3. V. K. Gurbani, A. Garvert, and J.D. Herbsleb, A Case Study of a Corporate Open Source Development Model, Proceeding of the 28th international Conference on Software Engineering ICSE '06, Shanghai, China, 472-481 (2006)

4. C. Rossi and A. Bonaccorsi, Why Profit-Oriented Companies Enter the OS Field? Intrinsic vs. Extrinsic Incentives. Proceedings of the fifth Workshop on Open Source Software Engineering, 1-5 (2005)

5. L. Dahlander and M. G. Magnusson, Relationships between Open Source Software Companies and Communities: Observations from Nordic Firms. Research Policy, 34(4), 481-493 (2005) 